\title{
Correlation between metabolic, liver profile, dietary habits and ultrasound scan determined non-alcoholic fatty liver disease changes in children aged 6- 18 years with body mass index
}

\author{
Minakshi Bansal ${ }^{1},{ }^{*}$ Rohit Vohra $^{1}$, Ashwani Sood ${ }^{1}$, Parveen Bhardwaj ${ }^{1}$ \\ Sri Lanka Journal of Child Health, 2018; 47(2): 125-128
}

\begin{abstract}
Introduction: Non-alcoholic fatty liver disease (NAFLD) is the commonest cause of liver disease worldwide and this is linked to the change in lifestyle and rise in prevalence of overweight and obesity among children.
\end{abstract}

Objectives: To study the correlation between metabolic, liver profile, dietary habits and ultrasound scan determined NAFLD changes in children aged 6-18 years old with their body mass index (BMI).

\begin{abstract}
Method: A tertiary care hospital based comparative, prospective study was conducted on 159 children aged 6-18 years at Indira Gandhi Medical College and Hospital, Shimla, India over a period of one year. Children presenting to the outpatient department (OPD) were assigned to two groups based on their BMI, group I with BMI of $\leq 85$ th centile and group II with BMI $>85$ th centile. Those with acute and chronic liver ailments and on drugs like vitamin E, statins or antihypertensives were excluded. These were subjected to venepuncture for estimating fasting blood sugar (FBS), liver function tests (LFTs) and complete lipid profile. Ultrasound scan was done to look for NAFLD changes.
\end{abstract}

Results: Children in group II had increased incidence of dyslipidaemia $93.7 \%$ vs $41.2 \%$, deranged LFTs $49.4 \%$ vs $12.5 \%$, increased FBS $10.1 \%$ vs $2.5 \%$ and NAFLD $40.5 \%$ vs $2.5 \%$ when compared to children in group I.

Conclusions: BMI and dietary habits have an important bearing on occurrence of NAFLD in children aged $6-18$ years old.

DOI: http://dx.doi.org/10.4038/sljch.v47i2.8477

${ }^{1}$ Indira Gandhi Medical College, India
${ }^{*}$ Correspondence: drrohitvohra87@gmail.com

(Received on 12June 2017: Accepted after revision on 28 July 2017)

The authors declare that there are no conflicts of interest

Personal funding was used for the project.

Open Access Article published under the Creative

Commons Attribution CC-BY (CC)
(Key words: NAFLD, BMI, dietary habits, metabolic profile, dyslipidaemia)

\section{Introduction}

Non-alcoholic fatty liver disease (NAFLD) is characterised by abnormal lipid deposition in hepatocytes in the absence of excess alcohol intake ${ }^{1}$. Obesity, type 2 diabetes, hypertension, and hypertriglyceridaemia are risk factors for NAFLD ${ }^{2}$ which is a hepatic manifestation of metabolic syndromes $^{3}$. During the last 20 years, prevalence of NAFLD has doubled probably due to the increased prevalence of overweight and obesity in the paediatric population worldwide ${ }^{4}$. Till now, NAFLD remained an incidental finding but looking at its consequences the American Academy of Pediatrics suggests biannual screening for liver disease with serum liver enzymes in obese and overweight 10 year old children with other risk factors ${ }^{5}$.

\section{Method}

This was a tertiary care hospital based prospective study done over a period of one year from $1^{\text {st }}$ June 2014 to $31^{\text {st }}$ May 2015 in children aged 6-18 years attending the outpatient department (OPD) of Indira Gandhi Medical College and Hospital, Shimla, India. Children with pathologic conditions involving the liver such as hepatitis B or hepatitis $\mathrm{C}$ virus infections, acute or chronic liver failure, cholestasis, metabolic diseases like alpha 1-antitrypsin deficiency, Wilson disease, diabetes mellitus, hypothyroidism, alcohol consumption, severe malnutrition, etc or on treatment with vitamin $\mathrm{E}$, statins, ursodeoxycholic acid, metformin, antihypertensives, valproate, prednisolone or methotrexate at the time of enrolment were excluded from the study.

The subjects of the study were enrolled randomly from children and adolescents of the age group 6-18 years attending the OPD and were assigned to each group based on their body mass index (BMI). Informed consent was taken from the parents or caregivers. The subjects were screened for obesity, deranged liver profile, dyslipidaemia and fatty liver changes by doing physical examination and relevant investigations.

Weight of the child was recorded on an electronic type of weighing scale, with minimal clothing and weight was measured to $\pm 0.1 \mathrm{~kg}$. Height was 
measured using a stadiometer to $\pm 0.1 \mathrm{~cm}$. BMI was measured by dividing the weight in kilograms by the square of the height in metres. After a thorough physical and systemic examination, the enrolled children were subjected to venepuncture under aseptic conditions for estimating fasting blood sugar, liver profile and lipid profile after overnight fasting for a minimum of 6 hours. An ultrasound hepatic scan was also performed in the study population to observe for evidence NAFLD.

The data thus collected were analysed using SPSS program for windows, version 17.0. Continuous variables are presented as mean $\pm \mathrm{SD}$, and categorical variables are presented as absolute numbers and percentages. Data were checked for normality before statistical analysis. Normally distributed continuous variables were compared using the unpaired t-test, whereas the MannWhitney $U$ test was used for those variables that were not normally distributed. Categorical variables were analysed using either the Chi square test or Fischer's exact test. For the two group comparisons, student t-test was used to evaluate the significance of the variables. For all statistical tests, a $p$ value less than 0.05 was considered significant.

\section{Results}

A total of 159 children aged 6-18 years qualified for inclusion in the study. They were then divided into two groups:

Group I: Study population with normal BMI comprising $80(50.3 \%)$ children

Group II: Study population with increased BMI comprising of $79(49.7 \%)$ children

Table 1 compares the consumption behaviour of junk food in both groups. Table 2 compares the liver profile in groups I and II. Table 3 compares the lipid profile in groups I and II. Table 4 compares the ultrasound scan (USS) determined fatty liver changes in groups I and II

Table 1: Comparison of consumption behaviour of junk food in groups I and II

\begin{tabular}{|c|c|c|c|c|c|}
\hline \multirow{3}{*}{ Consumption of junk food } & \multicolumn{4}{|c|}{ Body mass index (BMI) } & \multirow{3}{*}{$p$ value } \\
\hline & \multicolumn{2}{|c|}{ Group I $(n=80)$} & \multicolumn{2}{|c|}{ Group II $(n=79)$} & \\
\hline & Frequency & $\%$ & Frequency & $\%$ & \\
\hline Daily & 04 & 05.0 & 14 & 17.7 & 0.013 \\
\hline More than 2-3 times a week & 19 & 23.8 & 27 & 34.2 & 0.147 \\
\hline Once a week & 39 & 48.8 & 28 & 35.4 & 0.089 \\
\hline Once in 15 days & 12 & 15.0 & 09 & 11.4 & 0.502 \\
\hline Once a month & 06 & 07.5 & 01 & 01.3 & 0.117 \\
\hline
\end{tabular}

Table 2: Comparison of liver profiles in groups I and II

\begin{tabular}{|l|c|c|c|c|c|}
\hline \multirow{2}{*}{ Fasting liver profile } & \multicolumn{3}{|c|}{ Body mass index (BMI) } & \multirow{2}{*}{$\boldsymbol{p}$ value } \\
\cline { 2 - 5 } & \multicolumn{2}{|c|}{ Group I (n=80) } & Group II (n=79) & \% & \\
\cline { 2 - 5 } & Frequency & $\mathbf{\%}$ & Frequency & 50.6 & $<0.001$ \\
\hline Normal & 70 & 87.5 & 40 & 13.9 & 0.009 \\
\hline Raised aspartate transaminase & 02 & 02.5 & 11 & 22.8 & 0.089 \\
\hline Raised alanine transaminase & 10 & 12.5 & 18 & 39.2 & $<0.001$ \\
\hline Raised alkaline phosphatase & 01 & 01.3 & 31 & & \\
\hline
\end{tabular}

Table 3: Comparison of lipid profile of groups I and II

\begin{tabular}{|l|c|c|c|c|c|}
\hline \multirow{3}{*}{ Fasting lipid profile } & \multicolumn{3}{|c|}{ Body mass index (BMI) } & \multirow{2}{*}{$\boldsymbol{p}$ value } \\
\cline { 2 - 5 } & \multicolumn{2}{|c|}{ Group I (n=80) } & \multicolumn{2}{c|}{ Group II (n=79) } & \multirow{2}{*}{ \% } \\
\cline { 2 - 5 } & Frequency & Frequency & \% & \\
\hline Normal & 47 & 58.8 & 05 & 06.3 & $<0.001$ \\
\hline Cholesterol $>90$ th percentile & 02 & 02.5 & 13 & 16.5 & 0.003 \\
\hline Cholesterol $>95$ th percentile & 01 & 01.3 & 13 & 16.5 & 0.001 \\
\hline Triglycerides $>90^{\text {th }}$ percentile & 16 & 20.0 & 12 & 15.2 & 0.426 \\
\hline Triglycerides $>95^{\text {th }}$ percentile & 16 & 20.0 & 55 & 69.6 & $<0.001$ \\
\hline LDL $>90^{\text {th }}$ percentile & 01 & 01.3 & 06 & 07.6 & 0.064 \\
\hline LDL $>95^{\text {th }}$ percentile & 00 & 0.0 & 04 & 05.1 & 0.059 \\
\hline HDL $<10^{\text {th }}$ percentile & 04 & 05.0 & 12 & 15.2 & 0.038 \\
\hline HDL $<5^{\text {th }}$ percentile & 01 & 01.3 & 10 & 12.7 & \\
\hline
\end{tabular}

LDL: Low density lipoproteins; HDL: high density lipoproteins 
Table 4: Comparison of ultrasound scan determined fatty liver changes in groups I and II

\begin{tabular}{|c|c|c|c|c|c|}
\hline \multirow{3}{*}{ Ultrasound scan findings } & \multicolumn{4}{|c|}{ Body mass index (BMI) } & \multirow{3}{*}{$p$ value } \\
\hline & \multicolumn{2}{|c|}{ Group I (n=80) } & \multicolumn{2}{|c|}{ Group II $(n=79)$} & \\
\hline & Frequency & $\%$ & Frequency & $\%$ & \\
\hline Normal & 78 & 97.5 & 47 & 59.5 & $<0.001$ \\
\hline Grade 1 & 02 & 02.5 & 30 & 38.0 & $<0.001$ \\
\hline Grade 2 & 0 & 0 & 02 & 02.5 & 0.245 \\
\hline
\end{tabular}

Figure 1 shows the correlation of liver and lipid profile with fatty liver changes on ultrasound scan. Alanine transaminase (SGPT) values ranged from 13-139 with a mean \pm SD of $31.77 \pm 16.15$ in the subjects with normal USS findings as compared to $38.38 \pm 18.67$ with fatty liver changes, which is statistically significant with a $p$ value of 0.033 . Serum cholesterol, triglycerides, LDL and HDL levels in subjects with positive USS findings for fatty changes had mean \pm SD values of $185.68 \pm$ $33.13,189.53 \pm 48.02,99.39 \pm 26.11$ and $40.82 \pm$ 5.13 respectively. These results were statistically significant with a $p$ value of $<0.001$.

Table 5 shows the correlation of ultrasound scan findings with the consumption of junk food.

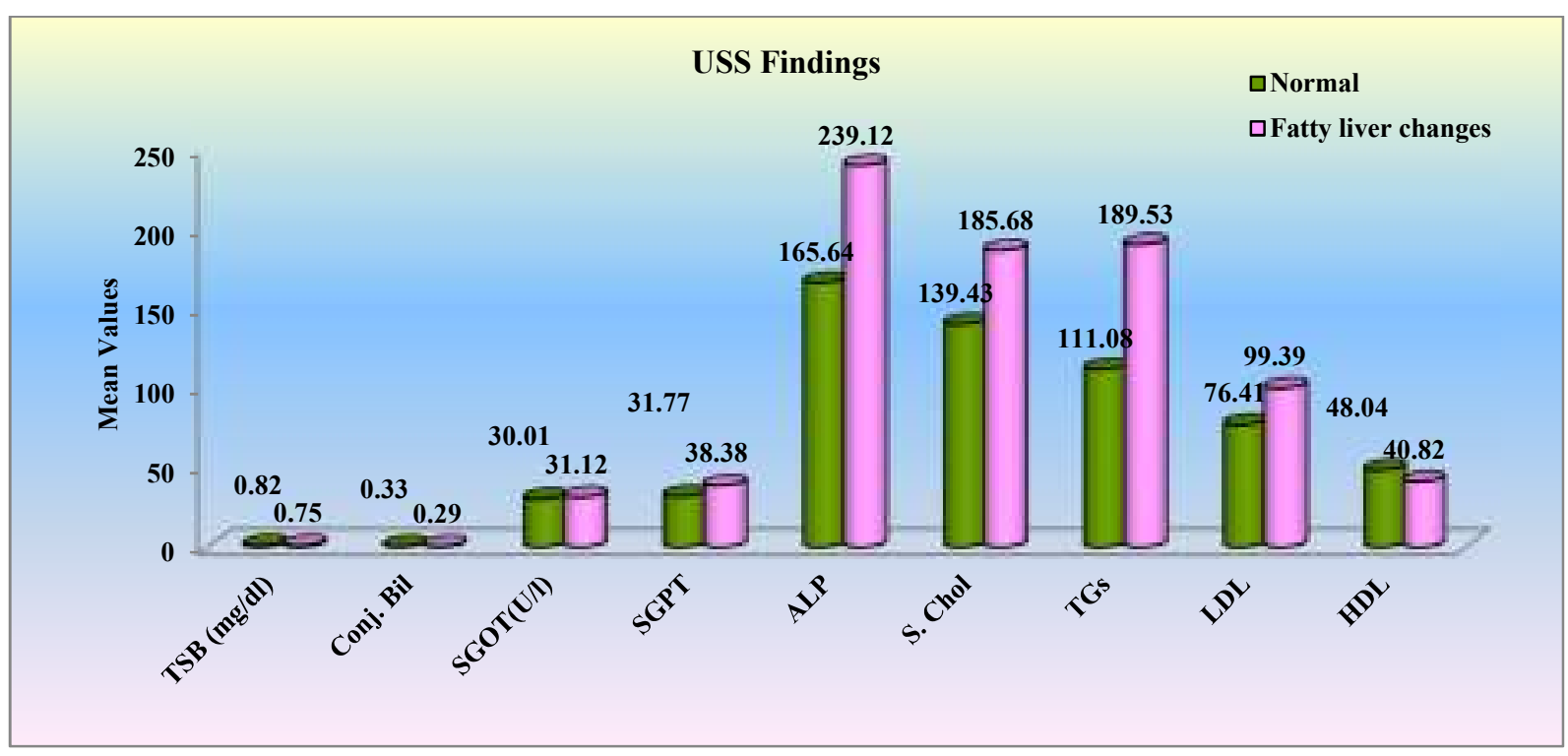

Figure 1: Correlation of liver and lipid profile with fatty liver findings on ultrasound scan

Table 5: Correlation of ultrasound scan findings with consumption of junk food

\begin{tabular}{|c|c|c|c|c|c|}
\hline \multirow{3}{*}{ Consumption of junk food } & \multicolumn{4}{|c|}{ Ultrasound scan findings } & \multirow{3}{*}{$p$ value } \\
\hline & \multicolumn{2}{|c|}{ Normal } & \multicolumn{2}{|c|}{ Fatty liver changes } & \\
\hline & Frequency & $\%$ & Frequency & $\%$ & \\
\hline Daily & 07 & 05.6 & 11 & 32.4 & $<0.001$ \\
\hline More than twice a week & 29 & 23.2 & 17 & 50.0 & 0.002 \\
\hline Once a week & 63 & 50.4 & 04 & 11.8 & $<0.001$ \\
\hline Once in 15 days & 20 & 16.0 & 01 & 02.9 & 0.048 \\
\hline Once a month & 06 & 04.8 & 01 & 02.9 & 1.000 \\
\hline Total & 125 & 100 & 34 & 100 & \\
\hline
\end{tabular}

\section{Discussion}

There is a phenomenal rise in the number of overweight and obese children worldwide. Association of NAFLD with obesity is now a well recognized entity even in children and NAFLD is increasingly being reported as a cause of chronic liver disease in children. Fatty liver changes on ultrasonography were found in $40.5 \%$ children in group II compared to $2.5 \%$ children in group I which is statistically significant $(\mathrm{p}<0.001)$. Parray et al also found increased prevalence of fatty liver disease in overweight and obese children ${ }^{6}$.

Raised aspartate transaminase, alanine transaminase (ALT) and alkaline phosphatase were seen in $13.9 \%$, $22.8 \%$ and $39.2 \%$ of subjects respectively with increased BMI as compared to only $2.5 \%, 12.5 \%$ and $1.3 \%$ of those with normal BMI $(p<0.001)$. Strauss et al reported that overweight and obese children were significantly more likely to have abnormal ALT levels compared with normal weight 
children $^{7}$. Tejeira et al also reported elevated serum ALT in $40 \%$ of overweight as compared to $4 \%$ of non-overweight children ${ }^{8}$.

Deranged lipid profile was seen in 73 out of 79 subjects in group II as compared to 33 of 80 children in group I. The participants with increased BMI had higher prevalence of hypercholesterolemia (cholesterol $>90$ th percentile, $16.5 \%, p=0.003$ ) and hypertriglyceridemia (triglycerides $>95$ th percentile, 69.6\%, $p<0.001$ ) and lower HDL (HDL $<10$ th percentile, $15.2 \%, \mathrm{p}=0.003$ ) levels as compared children with normal BMI. Plourde et al reported that overweight and obese children are at increased risk of dyslipidaemia as compared to normal children ${ }^{9}$.

In our study we found that children with increased BMI consumed more junk food and beverages when compared to children with normal BMI and were more likely to have fatty liver disease as determined by ultrasound $(p<0.001)$. A positive correlation was seen between lipid profile and SGPT levels and USS changes of fatty live disease in children of group II.

\section{Conclusions}

BMI and dietary habits have an important bearing on occurrence of NAFLD in children aged 6-18 years old.

\section{References}

1. Farell GC, Larter CZ. Non-alcoholic fatty liver disease; from steatosis to cirrhosis. Hepatology 2006; 43: S00-S112.

2. Angulo P. Non-alcoholic fatty liver disease. New England Journal of Medicine 2002; 346: 1221-31.

https://doi.org/10.1056/NEJMra011775

PMid: 11961152

3. Bugianesi E, McCullough AJ, Marchesini G. Insulin resistance: a metabolic pathway to chronic liver disease. Hepatology 2005; 42: $987-1000$. https://doi.org/10.1002/hep.20920

PMid: 16250043
4. Angulo P. Diagnosing steatohepatitis and predicting liver-related mortality in patients with NAFLD: two distinct concepts. Hepatology 2011; 53: 1792-4. https://doi.org/10.1002/hep.24403

PMid: 21557278

5. Barlow S E. "Expert Committee and Treatment of Child and Adolescent Overweight and Obesity: Expert Committee Recommendations Regarding the Prevention." Assessment, Report. Pediatrics 2007; 20.

6. Parray IA, Zargar SA, Khan BA, Ahmad B, Andrabi R, Kawoosa A, et al. Ultrasonographic prevalence of nonalcoholic fatty liver disease in Kashmir valley school children. International Journal of Science and Research 2013; 2(3): 299-301.

7. Strauss RS, Barlow S, Dietz WH. Prevalence of abnormal serum aminotransferase values in overweight and obese adolescents. Journal of Pediatrics 2000; 136(6):727-33.

https://doi.org/10.1067/mpd.2000.102940

PMid: 10839867

8. Quirós-Tejeira RE, Rivera CA, Ziba TT, Mehta N, Smith CW, Butte NF. Risk for non-alcoholic fatty liver disease in Hispanic youth with BMI \& gt; or $=95$ th percentile. Journal of Pediatric Gastroenterology and Nutrition 2007; 44:228-36.

https://doi.org/10.1097/MPG.0b013e3180 2d4acc

PMid: 17255837

9. Plourde G. Impact of obesity on glucose and lipid profiles in adolescents at different age groups in relation to adulthood. $B M C$ Family Practice 2002; 3: 18. https://doi.org/10.1186/1471-2296-3-18 PMid: 12379160 PMCid: PMC134463 